\title{
Asian Rhinoplasty
}

\author{
Samuel M. Lam, M.D., F.A.C.S. ${ }^{1}$
}

\begin{abstract}
Rhinoplasty for the Asian patient mandates an entirely different strategy than for the Occidental nose. The objective for Asian rhinoplasty is augmentation rather than reduction in which the dorsum is raised and the nasal tip projection and definition are enhanced. The watchword today is ethnic preservation rather than effacement for the Asian patient. An ethnically sensitive standard for aesthetic rhinoplasty for the Asian nose is proposed. Also, a thorough discussion of cultural issues that can be important when working with the Asian patient is undertaken to guide a surgeon through every phase of patient care. Finally, the technical details are elaborated on both rhinoplasty and revision rhinoplasty for the Asian nose with an emphasis on correcting previous silicone augmentation rhinoplasty, the most popular choice for material in the Far East but that has failed the author in achieving consistent results.
\end{abstract}

KEYWORDS: Asian, Oriental, nose, rhinoplasty, revision, silicone

Cosmetic enhancement of the Asian nose requires a unique philosophical approach and strategy in comparison with those necessary for Western rhinoplasty. First, cultural motivations may be entirely different. For instance, the Asian patient may desire rhinoplasty for no other reason than that a larger nose may impart a higher likelihood of accruing wealth. That provocative remark will be elaborated on in the following section. Second, the differences in cultural responses to cosmetic enhancement may be important to understand both for zero-generation Asian patients residing in the West and later generations who have become more assimilated into a Western perspective. Third, a culturally sensitive approach that underscores preservation but enhancement of ethnic identity may be critical in a good percentage of Asian patients seeking rhinoplasty. Cultural preservation has become the buzzword today for many Asians who desire cosmetic enhancement regardless of their global hemisphere of residence. Finally, the surgical technique for Asian rhinoplasty lies in diametric distinction to Western rhinoplasty, as the former is principally augmentative and the latter reductive in nature. This article will focus on both the cultural underpinnings that are mandatory in approaching the Asian patient as well as operative technique that can lead to a greater degree of consistency in aesthetic results for the Asian nose. A basic understanding of rhinoplasty technique is assumed and is not detailed herein except for advanced concepts that differ from Occidental rhinoplasty.

\section{CULTURAL ISSUES}

As mentioned, Asian rhinoplasty must be approached first by understanding the patient before understanding the operation. Many Western surgeons express dismay over dealing with the enigmatic Asian and fall into disbelief when a technically proficient result is interpreted as a failure by the Asian patient. Some of the larger folkloric beliefs that inform both Asians who live abroad and those who have recently immigrated to the West should be explored. As mentioned, Asians at times seek rhinoplasty principally or solely for the reason that they
${ }^{1}$ Willow Bend Wellness Center, Lam Facial Plastic Surgery Center \& Hair Restoration Institute, Plano, Texas.

Address for correspondence and reprint requests: Samuel M. Lam, M.D., F.A.C.S., Director, Willow Bend Wellness Center, Lam Facial Plastic Surgery Center \& Hair Restoration Institute, 6101 Chapel Hill Boulevard, Suite 101, Plano, TX 75093 (e-mail: drlam@ lamfacialplastics.com).
Cosmetic Surgery in the Ethnic Population: Special Considerations and Procedures; Guest Editor, Jamal M. Bullocks, M.D.

Semin Plast Surg 2009;23:215-222. Copyright (C) 2009 by Thieme Medical Publishers, Inc., 333 Seventh Avenue, New York, NY 10001, USA. Tel: +1(212) 584-4662.

DOI 10.1055/s-0029-1224801. ISSN 1535-2188. 
will have a greater predilection for wealth or fortune, as a larger proboscis may be thought to gain them that advantage. I recall a Chinese cook who attributed his recent pay hike directly to the effects of now sporting a larger nose. I also had a patient who is a secondgeneration Vietnamese in the United States whose fortune-teller informed him that a larger nose would help him attain a greater station in life. Of course, the surgeon must be careful in trying to perform a rhinoplasty when the motivation is exclusively or primarily informed by superstitious beliefs. I typically explore these thoughts more in depth for zero- or first-generation Asian patients who reside in the West to ensure that I can reliably achieve what they desire.

Other examples of folkloric beliefs of the face may be illustrative and impart further insight for the Western surgeon who desires to work on the Asian patient. Unlike the Caucasian patient who may find freckles to be an endearing feature, many Asians shun any kind of blemish and may also attribute negative life forces to these cutaneous lesions. Although reductive otoplasty may be common in the West, many Asians believe that larger ears may be a sign of wisdom that should not be curtailed in any way. Dimples-I create surgical dimples-can be deemed a cute facial feature but also be thought to increase one's marriage prospect and/or fertility. Exploring these complex and multilayered motivations may help establish and align realistic objectives with the patient.

Besides the folkloric factors enumerated, the desire for cosmetic rhinoplasty may be influenced by other ethnic and cultural factors. One of the top concerns that many Asians express about the procedure is whether they will retain their own identity and, above all, look natural, which in today's context follows the concept of ethnic preservation. Many Asians have a higher degree of comfort with me simply because I am Asian and therefore I would conceptually exercise more respect and attention to their native ethnicity. Likewise, I have had numerous African Americans feel that I would help them to achieve their aesthetic goals without violating their ethnicity compared with a Caucasian colleague. In general, I find that the height of the radix should fall roughly at the midpupil in most cases for Asians rather than the supratarsal crease, which may be a more appropriate dorsal height for white patients. A more conservative bridge height can achieve the desired softening of ethnic features while maintaining a more ethnically appropriate look that could look otherwise artificial if raised too high. The nasal tip contour may be softly augmented so that the rounded contour is maintained or more sculpted in appearance depending on the patient's desires and also what would fit the individual's features through artistic judgment. Divergent strategies to achieve these looks will be elaborated in a later section.
Using photographs of Asian models and reviewing a surgeon's "before-and-after" photographic results can be helpful to any prospective Asian patient. The caveat obviously would be twofold: the prospective patient may be more in love with the composite of the Asian model's face rather than the nose (which also would be unattainable) and further should be cautioned that looking at before-and-after results may be misleading in that every person's anatomy, especially for the nose, differs. Assuming that an individual is wholly desirous of maintaining ethnicity is also a bit erroneous. Today's globalized marketplace has stressed the beauty of models that are of mixed racial heritage. Also, with rising miscegenation, offspring from these mixed marriages may be striking a new ethnically blended aesthetic. Finally, I have noticed that the Asian who is partnered with a white individual oftentimes seeks procedures to soften ethnic features so that he or she may look more compatible with one's mate. Conversely, what I also find interesting is that many whites seek out Asians who have ethnic features precisely because they are more attracted to those attributes. Delving into these motivations can be an important part of any aesthetic consultation. However, whatever motivations make up the patient's desires, the surgeon should make a decision whether he or she should indulge every whimsy if doing so would ultimately lead to a perceptually artificial result. I myself do not bend too much from my aesthetic principles not out of fear that I would betray my ethnicity but more that I would create a result that would betray my aesthetic philosophy.

\section{USE OF SILICONE FOR AUGMENTATION RHINOPLASTY}

I think it is important to begin a discussion of augmentation rhinoplasty for the Asian nose with a thorough treatment of solid silicone implants because they remain the most popular option in the Far East for this procedure. If a surgeon ever decides to use solid silicone as an implant for the nose, the surgeon will almost definitely encounter patients who have had a silicone implant placed in the past who desire a revision procedure and the surgeon needs to know how to respond to questions regarding the use of that material. Personally, I advocated use of solid silicone in the past for the obvious reasons that the procedure was simple and reversible, accomplishable in the office setting under local anesthesia, and, if needed, removed in a similar fashion. Despite the Western surgeon's dismissal of silicone, I had a bias in favor of its use through my extensive training in the Far East. Over time, I became disenchanted with solid silicone for the reasons enumerated by Occidental surgeons, namely, the possible "shrink-wrapped" appearance to the nose, unpredictable cutaneous erythema that could fluctuate with 
environmental temperature changes, and an overall unnatural appearance.

I stopped using silicone for two principal reasons. First, no matter how thin I shaved the silicone down and no matter how soft a grade of silicone I used, the nasal bridge through encapsulation would ultimately appear too high after a few months to years when encapsulation of the silicone inevitably occurred. Second, I found an unacceptable incidence of distal migration, in which the implant would press downward on the nasal tip and risk pressure necrosis if not frank extrusion. I believe the weight of the silicone poses the greatest problem over time.

Because of my experience with silicone, I have become more sensitive about how to handle problems that should arise with a patient who already has a silicone implant. Oftentimes, the first instinct is to remove the implant and replace it with a more acceptable dorsal augmentation graft, like expanded polytetrafluoroethylene (ePTFE; to be discussed) or an autograft like cartilage. However, most silicone implants that are placed appear too high in the nasal bridge to begin with. I have found that once the implant is removed, the resulting scarring and encapsulation that ensue after the initial rhinoplasty is oftentimes adequate to achieve a natural dorsal height. Sometimes, placing another dorsal implant no matter how thin can return the patient back to the undesirable position of having a bridge that is too high.

Removal of a silicone implant can be performed easily in the office setting with local anesthesia. For a right-handed surgeon, an abbreviated marginal incision can be made in the right nostril to access the implant. Local anesthesia can be administered simply to the nasal tip near the marginal incision without having to infiltrate all the way up the nasal dorsum. The injection is performed slowly without moving the needle (an 0.5-inch, 30-gauge) and permitting the anesthetic to spread to limit any potential discomfort. A curved Mosquito clamp can be used to remove the implant by using the tines for palpation of the implant. It is important that the implant be firmly grasped with the tines and slowly removed under deliberate traction to minimize shearing the implant into pieces.

If there is any suspicion of calcification around the silicone implant (which can happen), the surgeon might consider performing the procedure under limited sedation, as the calcification can be a tenacious barrier for easy implant removal. The best indicator of calcification is palpation and observation of the nasal dorsum for signs of irregularities that should not be apparent with a smooth implant, especially if these irregularities occurred years after rhinoplasty. At times, these calcifications if they are not completely removed will mandate an external rhinoplasty approach to remove them in a piecemeal fashion, which still may not be completely possible.

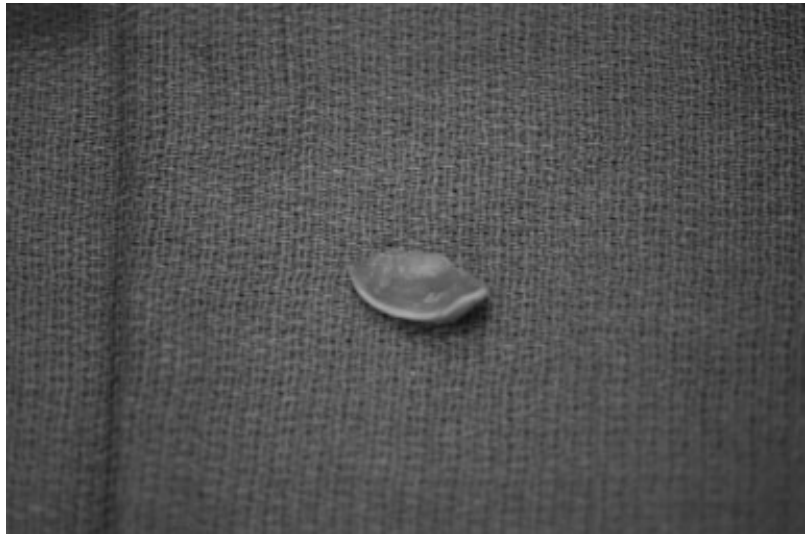

Figure 1 The cymba concha cartilage is shown removed as an intact single piece to be used to reconstruct the lower lateral cartilage by Pedroza's method, which he calls the "seagull" graft.

Another limitation with previous augmentation rhinoplasty using a silicone implant is the risk of pressure necrosis on the native dorsum and particularly the nasal tip cartilages. A very large L-shaped implant can cause these malformations over time. A patient should be alerted to this possible outcome and be counseled on the need for a formal revision rhinoplasty in that case. If simple silicone extraction fails to accomplish the task at hand, a formal revision rhinoplasty procedure may be needed once the implant is removed. One of the most devastating complications after Asian rhinoplasty is the foreshortened or contracted nasal tip, in which the pressure of the silicone (now removed) causes the nasal-tip skin to ride upwards and contract the nose into a porcine appearance. Revision rhinoplasty in this case is much more difficult and can be accomplished through use of extended spreader grafts to lengthen the nose again as well as possibly a low, wide inverted- $\mathrm{V}$ columellar incision at the columellar-labial angle to recruit lip tissue up to the nose as needed.

Another strategy that can be used instead of or in combination with an extender spreader graft to rebuild a partially deformed nasal tip is the seagull graft popularized by Dr. Fernando Pedroza. This technique employs the cymba concha of one ear (Fig. 1) divided lengthwise into two equal halves (Fig. 2) then trimmed so that one end resembles the smaller native medial crus and the other end resembles the wider lateral crus (Fig. 3). A 5-0 Vicryl (Ethicon, West Somerville, NJ) suture is used as a mattress to bend one end of each cartilage piece to resemble the natural curvature of the medial and lateral crus (Figs. 4 and 5). The two pieces are sutured together (Fig. 6) to create an entirely new lower lateral cartilage to be used in an onlay fashion over the partially attenuated native cartilages (Fig. 7). 


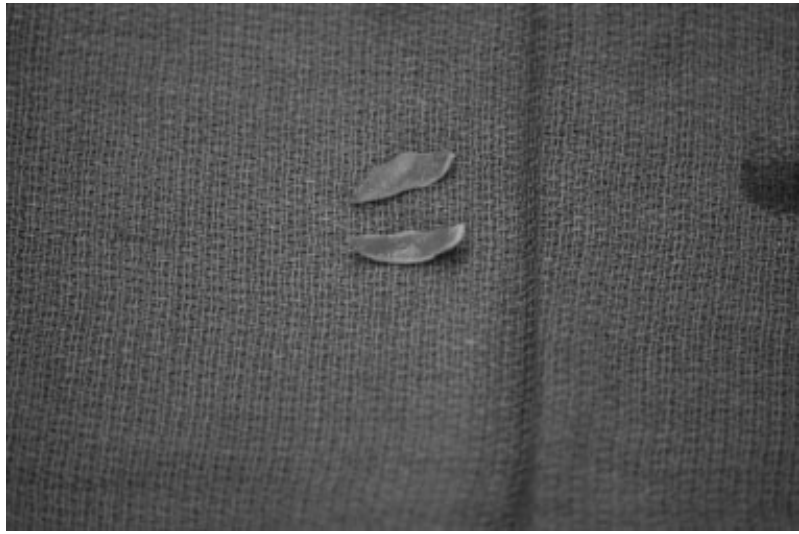

Figure 2 The conchal cartilage is then divided lengthwise into approximately equal halves.

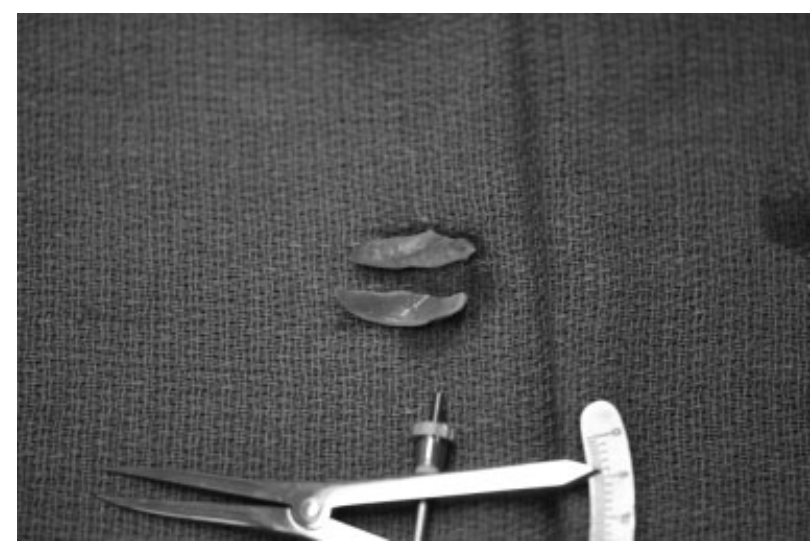

Figure 3 The two pieces are then trimmed so that they are of equal size and so that one narrower end resembles the medial crus. A caliper is used to ensure accurate measurements for symmetry.

\section{MY STRATEGY FOR ASIAN RHINOPLASTY}

Although completely autogenous, Asian rhinoplasty can be undertaken successfully using exclusively septal, auricular, and/or costal cartilage grafting. I prefer using a combination of alloplastic and autogenous rhinoplasty.

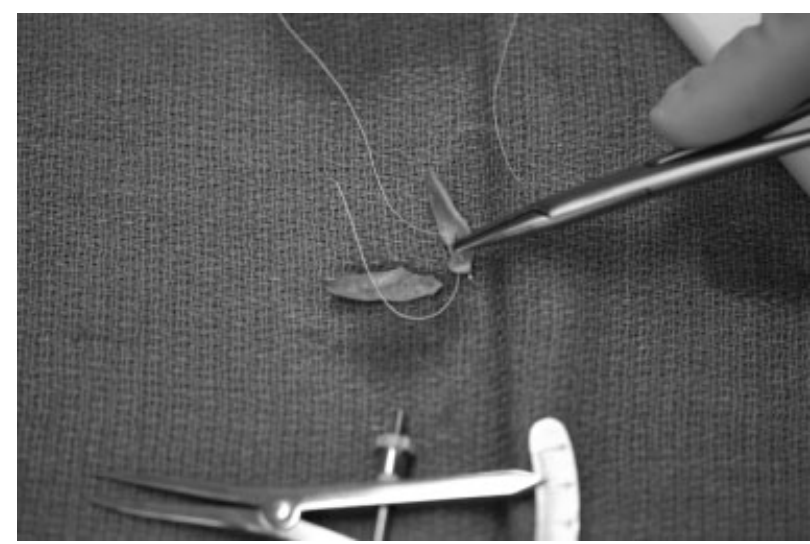

Figure 4 A 5-0 Vicryl suture is used as a mattress to bend the narrower end over to create the new dome.

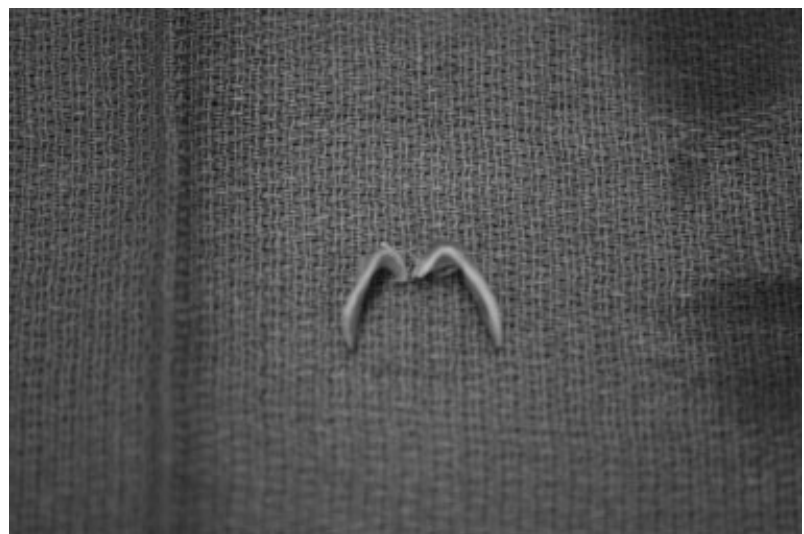

Figure 5 The two sutured pieces are shown adjacent to each other in the configuration that they will assume as the new lower lateral cartilage replacements.

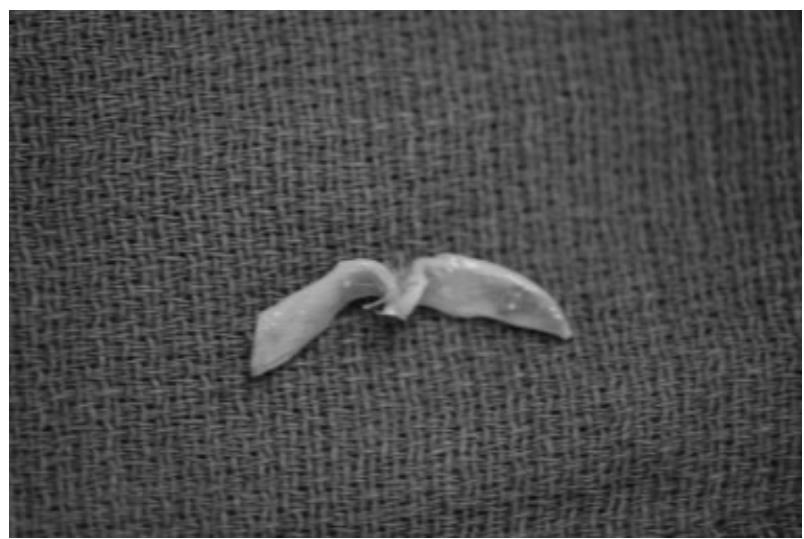

Figure 6 The medial crura are then sutured together to form the natural joined arc of the newly fashioned lower lateral cartilages.

The trend in the past few years has been to move away from using solid silicone implants by many experienced practitioners in the Far East (and in the West) as well as a move toward what has been termed "two-piece" augmentation in which the dorsum and the tip are augmented

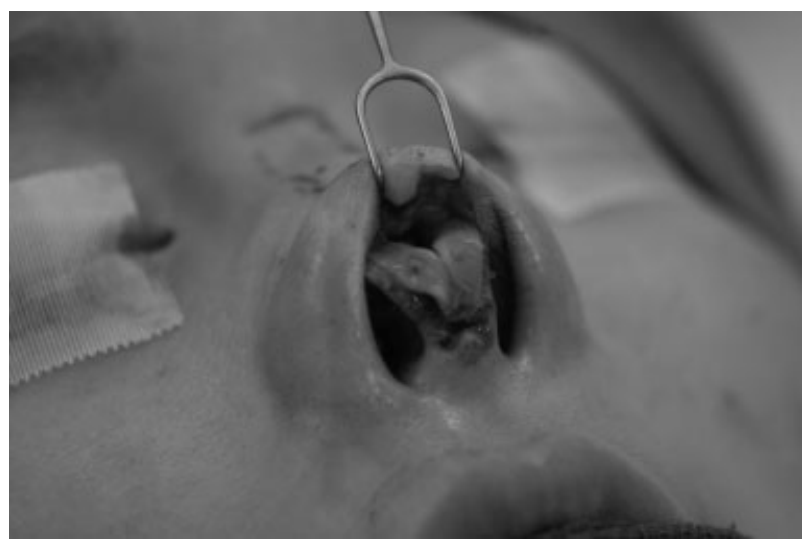

Figure 7 The graft is shown sutured as an onlay over the existing lower lateral cartilages. 
separately. I favor a two-piece augmentation with solid ePTFE 1- and 2-mm sheets for the dorsum and septal and/or auricular cartilage for the nasal tip. Now that ePTFE is no longer being manufactured by W.L. Gore for the cosmetic market, other manufacturers have stepped into the vacuum to alleviate this deficiency (Surgiform Technology, Ltd., Columbia, SC). In general, I prefer use of the sheets rather than the solid blocks, as I find the sheets of 1- to 3-mm thickness to be sufficient to augment most Asian noses. In addition, they conform more readily to the shape and contour of the native nasal bridge compared with even carved solid blocks. In any case, I use a needle driver to crimp the lateral $1 \mathrm{~mm}$ of the ePTFE sheet to provide a smoother transition over to the unaugmented lateral borders. I also trim the inferior aspect into a triangular dart, which is ensconced between the medial crura and sutured with 5-0 polydioxanone (PDS [Ethicon, West Somerville, NJ]) suture to limit distal migration. I believe that the lighter weight to GoreTex (ePTFE; W.L. Gore \& Associates, Flagstaff, AZ) and not using it to augment the tip as a single unit help to limit the problem of distal migration. All of my Asian rhinoplasties are performed via an external rhinoplasty approach with direct visualization of the ePTFE implant during insertion and shortly thereafter. It is easy inadvertently to crimp and fold the edges of the ePTFE implant if performed without deliberate intention and careful inspection. Just like a silicone implant, the implant is better affixed to the nasal dorsum through shearing the periosteum of the nasal bones with a periosteal elevator before inserting the implant. Also, irrigation with antibiotic-soaked saline solution can help reduce the risk of infection postoperatively and improve visualizing the placement of the implant under direct vision with a Converse retractor. Palpating the dorsum a final time with moistened gloved fingers before addressing the nasal tip can help provide additional reassurance that the implant is not malpositioned.

Of note, I do not believe that osteotomies are indicated with Asian rhinoplasty for many reasons. First, they can destabilize the position of the nasal implant if placed before the implant is inserted. Worse, the implant can be completely malpositioned by the osteotome if done after the implant is already placed. Third, the narrowed dorsum can make the nasal tip, which is typically too broad, look even broader due to a relative reduction in width. For all of these reasons, osteotomies in the Asian nose when performing augmentation rhinoplasty is both unnecessary and perhaps detrimental. The way in which a dorsal onlay graft creates a narrower appearance to the bridge is by placing the implant so that it does not extend all the way across the dorsum from lateral nasal wall to lateral nasal wall but about two thirds of the dorsal width so that it completes the cap to the pyramid and thereby creates the illusion of narrowness without having to narrow a broad dorsum.
As far as the nasal tip is concerned, I prefer the use of septal cartilage but at times require the use of auricular cartilage due to the scant supply and thinness of septal cartilage in the Asian patient. I almost always start with septal cartilage if available, as it provides a wonderful columellar strut to maintain tip projection and also to minimize donor-site morbidity. However, if a patient has a relatively amorphous and poorly projected nasal tip, use of both septal and one auricular conchal cartilage may be required to attain the desired degree of tip projection and contour. If I am trying to maintain a more natural, rounded configuration of the nasal tip, onlay grafts with septal and/or auricular cartilages shaped into an oval and laid down horizontally across the domes can provide increased tip projection without adding significant sharpness to the nasal tip contour (Fig. 8). If the patient desires a more sculpted nasal appearance, I prefer a shield graft with a possible cap graft (below the anterior edge of the shield graft) and/or blocking graft (placed just above along the anterior edge of the shield graft resting against the native domes) to limit undesirable tip rotation. A shield graft also will help increase a deficient and retruded premaxilla as a side benefit (Fig. 9). Conversely, I almost never use shield grafts in Caucasian patients whose relatively thinner skin can reveal the tombstone appearance of the graft after a few years time. Defatting the skin in the supratip region can also help to achieve better tip definition as desired.

The width of the nasal base is another concern for many Asian patients. A typical dome-binding suture should only be performed in the Asian patient with an underrotated nasal tip without necessarily a very broad aspect to the nasal base. The reason for this fear of performing a dome-binding suture is that rotation of the nasal tip can lead to greater nostril show in a wide nasal base that may be deemed unaesthetic. In addition, a cultural impression that was not outlined earlier is that many Asians believe that nostril show from an anterior view of the individual can be synonymous with the risk of money flooding outward (through the implied holes of the nose). In fact, some Asian patients express motivation for Asian rhinoplasty simply to cut down the possible loss of money through these enlarged portals. Another important pearl when evaluating an Asian patient for cosmetic rhinoplasty is the relative width of the nasal lobule vis-à-vis the alae. If the alae are relatively narrow compared with the nasal lobule, the lobule can look more bulbous when the alae are reduced. The simple way to demonstrate that to a patient is to pinch gently the alar sidewalls and show the patient the resulting bulbous nature of the nasal tip. If the nasal alae are relatively wider than the nasal lobule, then an alarbase reduction can be performed. I prefer to perform a Sheen flap in which a narrow bridge of tissue is preserved medially so that the natural curvature of the nasal sill is not violated. Additional reduction of the nostril size can 
A
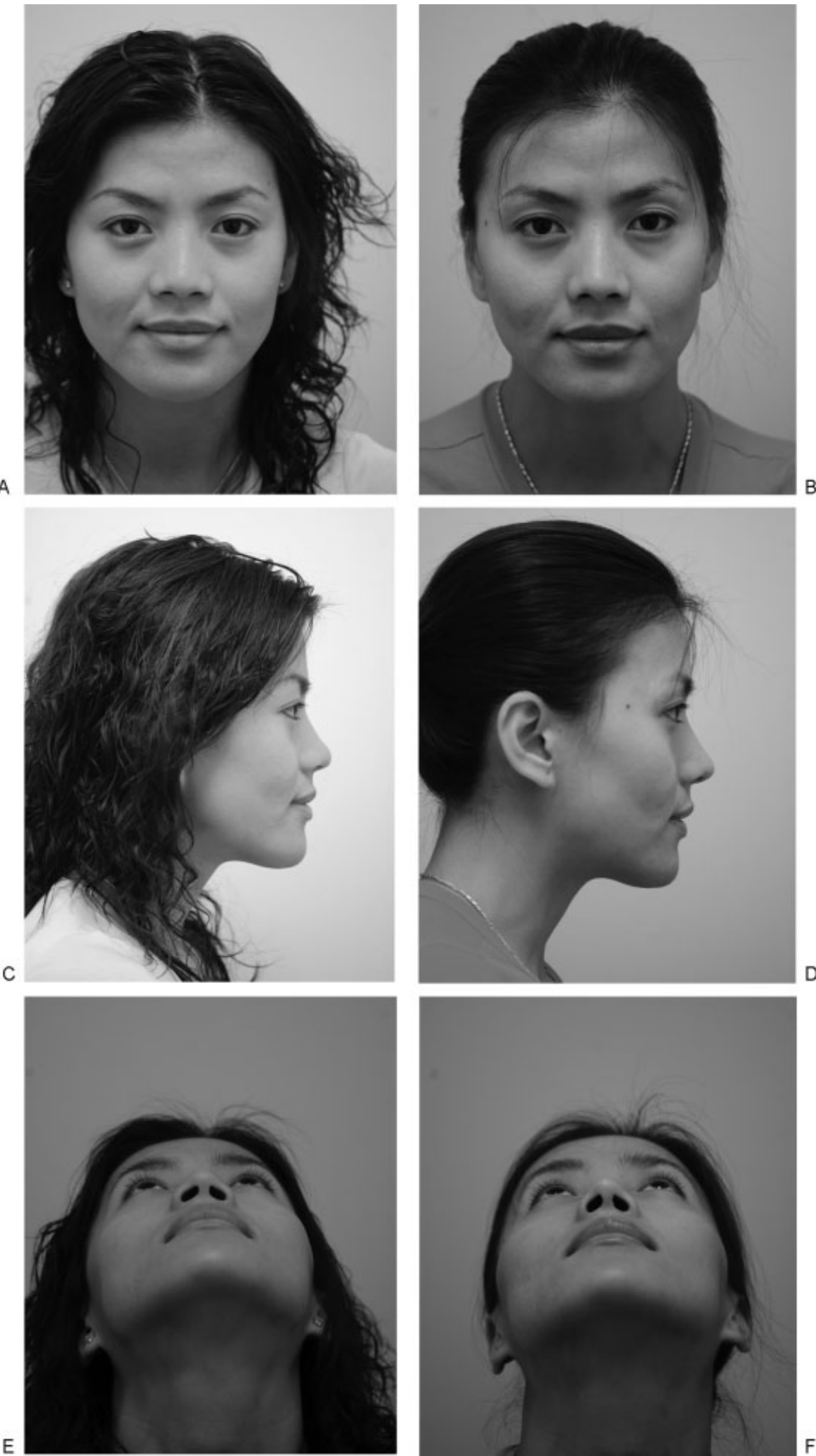

Figure 8 The patient is shown $(A, C, E)$ before and (B, D, F) after external rhinoplasty that involved a single ply of 1-mm ePTFE sheet for the nasal dorsum, a one-layered septal cartilage onlay graft, and a septal columellar strut that was positioned lower than her natural medial crura to extend her columella inferiorly. She also had a nasal sill reduction. 

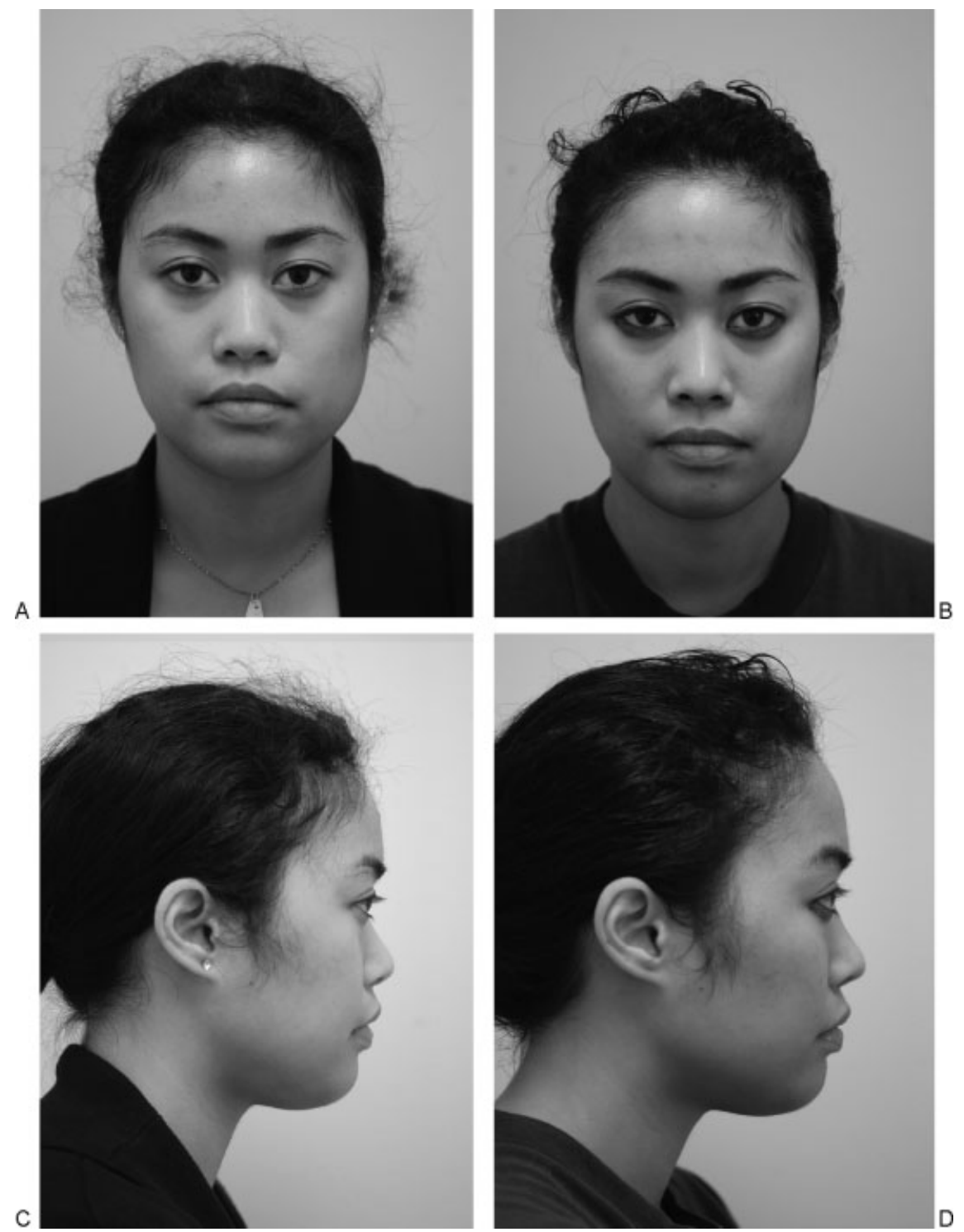

Figure 9 This patient is shown $(A, C)$ before and $(B, D)$ after external rhinoplasty and full facial fat transfer. She had a shield graft and single-layered blocking graft placed to her tip and a two-ply of 1-mm ePTFE sheet to the dorsum. She had fat transferred to her brow, anterior cheek, lateral cheek, and anterior chin to narrow the shape of her face and to provide more dimensionality to it.

be performed using a separate nasal sill reduction. However, I have found that an isolated nasal sill reduction causes unintended further rounding of the ala, making a narrower ala actually at times look wider. This is not the case if the nostril sill is the principal problem with a very wide distance, as in some African American noses. In this case, an isolated nasal sill reduction can be undertaken. The patient should also be counseled on the possible need for a protracted period of time in which the alar-base scar is visible with also the probability that the scar need be refined with mechanical dermabrasion. This limitation is very real and should be part of every preoperative consultation with the patient, especially the Asian patient in which an incision can turn erythematous, hypertrophic, hyperpigmented, and hypopigmented for what may seem an intractably long time. Understanding the aesthetic balance between the dorsum, the lobule, and the alae and the effect that reducing or enlarging one structure will have on the appearance of the other two is particularly critical when dealing with the Asian nose.

\section{CONCLUSION}

Asian rhinoplasty requires an entirely different philosophical approach and surgical armamentarium than that for an Occidental counterpart. Hopefully, this brief article has helped elucidate some of the fundamental aspects of cultural biases, ethnic sensitivity, and technical execution that are inherent features of any successful 
cosmetic rhinoplasty endeavor, whether primary or revision, for the Asian individual.

\section{SUGGESTED READINGS}

McCurdy JA Jr, Lam SM. Cosmetic Surgery of the Asian Face. 2nd ed. New York, NY: Thieme Medical Publishers; 2005

Lam SM. Aesthetic facial surgery for the Asian male. Facial Plast Surg 2005;21:317-323

Pedroza F, Anjos GC, Patrocinio LG, Barreto JM, Cortes J, Quessep SH. Seagull wing graft: a technique for the replacement of lower lateral cartilages. Arch Facial Plast Surg 2006;8:396-403
Jung DH, Moon HJ, Choi SH, Lam SM. Secondary rhinoplasty of the Asian nose: correction of the contracted nose. Aesthetic Plast Surg 2004;28:1-7

Lam SM, Kim YK. Augmentation rhinoplasty of the Asian nose with the "bird" silicone implant. Ann Plast Surg 2003;51:249256

Shirakabe Y, Suzuki Y, Lam SM. A new paradigm for the aging Asian face. Aesthetic Plast Surg 2003;27:397-402

Jung DH, Choi SH, Moon HJ, Chung IH, Im JH, Lam SM. A cadaveric analysis of the ideal costal cartilage graft for Asian rhinoplasty. Plast Reconstr Surg 2004;114:545550 